\title{
Características demográficas, morbilidad y mortalidad de los pacientes adultos con neutropenia febril tratados con cefepima
}

\author{
Gerardo A. Puentes, Camilo Quiroga, Carlos A. Álvarez, José R. Támara y Álvaro Ruiz
}

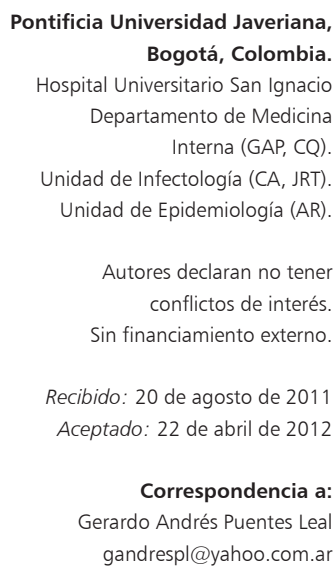

Pontificia Universidad Javeriana,

Bogotá, Colombia.

Hospital Universitario San Ignacio Departamento de Medicina Interna (GAP, CQ)

Unidad de Infectología (CA, JRT) Unidad de Epidemiología (AR)

Autores declaran no tener conflictos de interés Sin financiamiento externo.

Recibido: 20 de agosto de 2011 Aceptado: 22 de abril de 2012

Correspondencia a: Gerardo Andrés Puentes Leal gandrespl@yahoo.com.ar

\section{Demographics, morbidity, and mortality febrile neutropenic adult patients treated with cefepime}

Introduction: Cefepime efficacy for treatment of febrile neutropenia (FN) in cancer adult patients is a controversial issue. Objective: To describe the demographic characteristics and general mortality of patients suffering from febrile neutropenia treated with cefepime in a fourth-level Latin American hospital. Patients and Methods: A cross-sectional observational study was performed. Study settled at San Ignacio of Bogotá, Colombia. University Hospital from January 2004 to December 2008. Results: A total of 333 patients were treated with cefepime, of whom 125 had suffered FN and met pre established inclusion and exclusion criteria. The general mortality was $14.4 \%$, which was similar to the overall mortality in FN in other reports. Conclusions: Although there is still no clarity regarding the efficacy of cefepime in FN, its use has not been restricted. This study did not identify an excess risk of mortality in patients treated with cefepime.

Key words: neutropenia, mortality, cefepime, cephalosporins.

Palabras clave: neutropenia, mortalidad, cefepima, cefalosporina.

\section{Introducción}

L a neutropenia febril (NF) es una condición clínica frecuentemente encontrada en pacientes con cáncer sometidos a quimioterapia, al igual que en otras causas de inmunosupresión ${ }^{1,2}$. Más de $50 \%$ de los pacientes con neutropenia que presentan fiebre tienen infección oculta ${ }^{2}$. Aquellos pacientes con infección oculta no tratada, cursan rápidamente a deterioro y alto riesgo de mortalidad, motivo por el cual está indicado el inicio de terapia antimicrobiana empírica de amplio espectro ${ }^{2-7}$. Varios estudios han demostrado que la monoterapia es equivalente a la terapia combinada, siendo los agentes autorizados como monoterapia empírica cefalosporinas de tercera generación (ceftazidima), cefalosporinas de cuarta generación (cefepima), piperacilina/tazobactam o carbapenémicos (imipenem/cilastatina, meropenem) $)^{1,2,8-15}$.

La terapia empírica de primera línea usada en nuestra Institución es cefepima; sin embargo, recientemente se han publicado tres meta-análisis. El primero, publicado en The Journal of Antimicrobial Chemotherapy en 2006, donde se analizan 17 estudios encontrando aumento de la mortalidad en los pacientes tratados con cefepima en $44 \%$ (RR: 1,44, 95\% CI 1,06-1,94; $\mathrm{p}=0,02)^{16}$. Un segundo meta-análisis publicado en Lancet Infectious Diseases en mayo de 2007, analiza el uso de cefepima comparado con otros -lactámicos, carbapenémicos y piperacilina/ tazobactam incluyendo en total 57 estudios en los cuales se evidenció un exceso de mortalidad en los pacientes tratados con cefepima en un rango entre 8 y $49 \%$ con un promedio de $26 \%$ (la proporción de riesgo [RR] 1,26 [95\%
CI1,08-1,49]); adicionalmente en los desenlaces secundarios no hubo diferencias estadísticamente significativas al confrontar cefepima con los otros comparadores, tanto en el análisis global como en los subgrupos: fracaso clínico (RR 0,98. IC 0,93-1,03), fracaso microbiológico (RR 0,92, IC 0,84-1,02), super-infecciones (RR 0,96. IC 0,79-1,17) y eventos adversos (RR 0,99. IC 0,94-1,04). La excepción fue la comparación de cefepima y piperacilina/tazobactam con respecto al fracaso clínico (RR 1,09. IC 1,01-1,18; $\mathrm{p}=0,04)^{17,18}$. Un tercer meta-análisis publicado por The Cochrane Collaboration en 2010, donde se analizan 21 estudios, evidenció una mortalidad significativamente superior con el uso de cefepima comparándose con carbapenémicos, ceftazidima y piperacilina/tazobactam (RR 1,39. IC 1,04-1,86) ${ }^{19}$. Finalmente, la US Food and Drug Administration (FDA) realizó un meta-análisis publicado en Clinical Infectious diseases en 2010, que evalúo la eficacia y seguridad de cefepima en sus diferentes indicaciones ${ }^{20}$ abarcando también el subgrupo de NF, en el cual no se encontró aumento estadísticamente significativa en la mortalidad al usar cefepima ${ }^{21,22}$. De la misma forma, en las guías de la Sociedad Americana de Enfermedades Infecciosas (IDSA) para el manejo de pacientes neutropénicos con cáncer, publicadas en 2010, se recomienda el uso de cefepima como monoterapia para el tratamiento de esta entidad ${ }^{22,23}$.

En nuestra institución existe un gran número de pacientes que ingresaron con NF a quienes ya se administró cefepima siguiendo el protocolo de manejo. Desde el año 2001 se incluyó como primera elección, en monoterapia empírica, el uso de cefepima y en condiciones 
seleccionadas como es la sospecha de foco infeccioso intra-abdominal piperacilina/tazobactam y recientemente carbapenémicos previo al uso de -lactámicos o quinolonas. De forma rutinaria no utilizamos terapia combinada con aminoglucósidos y para la prescripción de vancomicina se siguen las indicaciones establecidas en las guías de manejo de IDSA.

Dada la reciente controversia descrita con el uso de cefepima, especialmente en pacientes con NF, el objetivo de este estudio es describir la experiencia en nuestra institución en el manejo de la NF tratada con cefepima y su mortalidad asociada.

\section{Materiales y Métodos}

Se realizó un estudio observacional, descriptivo, de corte transversal, que incluyó a todos los pacientes con diagnóstico de NF internados en el Hospital Universitario San Ignacio, tanto en sala general como en la unidad de cuidados intensivos, que fueron tratados con cefepima en el período entre enero de 2004 y diciembre de 2008. Los criterios de inclusión fueron: edad sobre 16 años, diagnóstico de NF, según la definición de las guías americanas de NF (recuento absoluto de neutrófilos $<500$ céls $/ \mathrm{mm}^{3}$ y aquellos con recuento $<1.000$ céls $/ \mathrm{mm}^{3}$, en los cuales era previsible su posterior disminución a menos de 500 céls $/ \mathrm{mm}^{3}$ en las siguientes 48 horas); presencia de fiebre cuantificada mayor de $38,3^{\circ} \mathrm{C}^{24}$ y en quienes su manejo antimicrobiano inicial fue con cefepima a una dosis de 3 a 6 g/día. Se excluyó a quienes tenían uso previo de antimicrobianos de amplio espectro diferentes a cefepima en los 15 días antes del episodio de NF y uso de cefepima por un tiempo menor a 72 horas. No se excluyó el uso concomitante de antivirales o antimicóticos. Como desenlace a evaluar se establecieron la identificación de foco infeccioso al final del tratamiento documentado por manifestación clínica y por exámenes de laboratorio, reducción o ampliación del espectro antimicrobiano de acuerdo al antibiograma, falla terapéutica definida como suspensión o cambio de la terapia antimicrobiana debido al deterioro clínico del paciente y como desenlace final, muerte al final de la hospitalización.

Se hizo una descripción demográfica con frecuencias de tendencia central y de dispersión de las variables clínicas relevantes descritas en la literatura médica (datos tomados directamente de la historia clínica de los pacientes) y se evaluaron asociaciones entre las variables demográficas y los desenlaces, especialmente entre los pacientes que fallecieron y los que sobrevivieron. Se utilizó el programa STATA 10.0 (Data Analysis and Statistical Software, USA). Se utilizó ${ }^{2}$ de Pearson para establecer la significancia de las variables cualitativas y la prueba $t$ de Student para las variables continuas. Se definió significación estadística mediante un valor de $\mathrm{p}<0,05$.

\section{Resultados}

Se identificaron 333 historias clínicas con uso de cefepima. Se categorizaron y tabularon por años, identificándose 125 eventos de NF que cumplían los criterios de inclusión. Se excluyó un paciente por tener otro antimicrobiano diferente a cefepima dentro de los 15 días previos al diagnóstico de NF. En la Tabla 1 se describen las variables recolectadas.

De todas las NF en tratamiento con cefepima, 32,8\% tenían una o más co-morbilidades de distribución muy heterogénea y $96 \%$ cursaban con un proceso neoplásico.

Como desenlace al culminar el tratamiento se encontró que en $36 \%$ no se confirmó un proceso infeccioso, y de $64 \%$ de las infecciones documentadas, en $8 \%$ se redujo el espectro antimicrobiano y en $2,4 \%$ se aumentó. En $24 \%$ se consideró falla terapéutica y la mortalidad global de todos los pacientes con NF en tratamiento con cefepima fue de $14,4 \%$.

Tabla 1. Características demográficas de 125 pacientes con neutropenia febril tratados con cefepima

\begin{tabular}{|c|c|c|}
\hline Características & $\mathbf{n}$ & $\%$ \\
\hline Edad & $36,39 \pm 16,6$ & \\
\hline Sexo masculino & 70 & 56 \\
\hline Neoplasia & 120 & 96 \\
\hline Tumores hematológicos & 95 & 79,2 \\
\hline Tumores sólidos & 25 & 20,8 \\
\hline Infección por virus de inmunodeficiencia humana & 3 & 2.4 \\
\hline Trasplante & 2 & 1,6 \\
\hline Cardiaco & 1 & 50 \\
\hline De precursor hematopoyético & 1 & 50 \\
\hline Uso de inmunosupresor & 112 & 89,6 \\
\hline Catéter venoso central & 14 & 11,2 \\
\hline Uso de factor estimulante de crecimiento de colonias & 24 & 19,2 \\
\hline Profilaxis antimicrobiana & 5 & 4 \\
\hline Neutrófilos absolutos (céls /mm³) & $222 \pm 213,30$ & \\
\hline Días de neutropenia & $9,56 \pm 7,67$ & \\
\hline Cultivos positivos & 84 & 25 \\
\hline Co-morbilidades & 43 & 32,8 \\
\hline Duración de antibioticoterapia con cefepima (días) & $8,28 \pm 3,90$ & \\
\hline Uso concomitante de antimicrobianos & 73 & 58,4 \\
\hline Fin de tratamiento sin foco infeccioso identificado & 45 & 36 \\
\hline Fin de tratamiento con infeccion documentada & 80 & 64 \\
\hline Aumento del espectro antimicrobiano por antibiograma & 10 & 8 \\
\hline Reducción de antimicrobiano por antibiograma & 3 & 2,4 \\
\hline Falla terapéutica & 30 & 24 \\
\hline Fallecimientos & 18 & 14,4 \\
\hline
\end{tabular}




\section{Focos infecciosos}

La distribución de los focos infecciosos de acuerdo al sitio anatómico, documentados en orden de frecuencia fueron: bacteriemia $(17,6 \%)$, procesos infecciosos de piel y tejidos blandos $(13,6 \%)$, sistema respiratorio $(11,2 \%)$, gastrointestinal $(8 \%)$, tracto urinario $(2,4 \%)$, sistema nervioso central $(0,8 \%)$, otros $(10,4 \%)$.

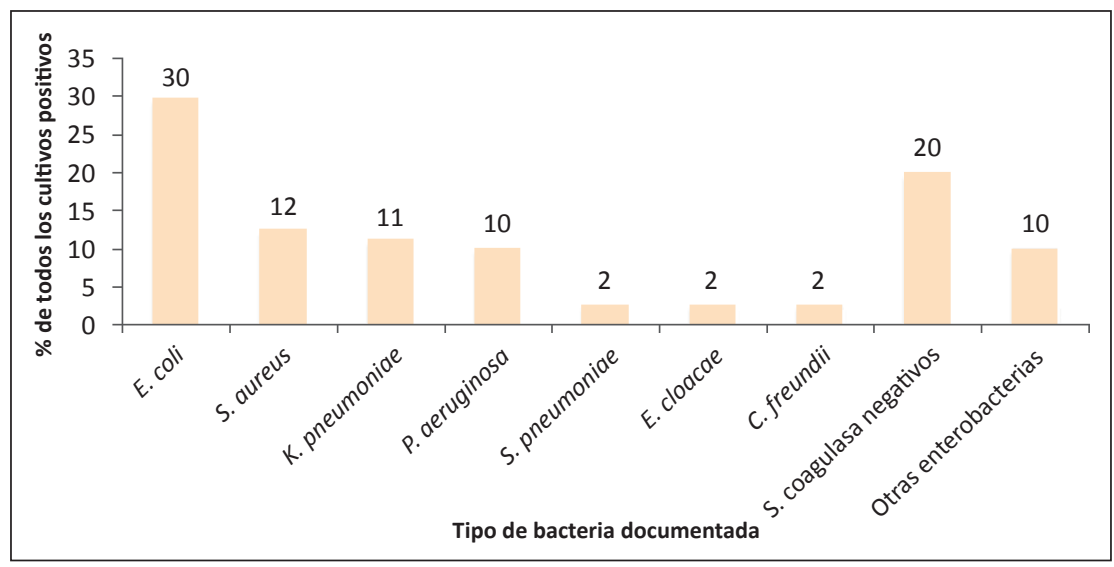

Figura 1. Frecuencia de las especies bacterianas aisladas en 125 pacientes con neutropenia febril tratados con cefepima.

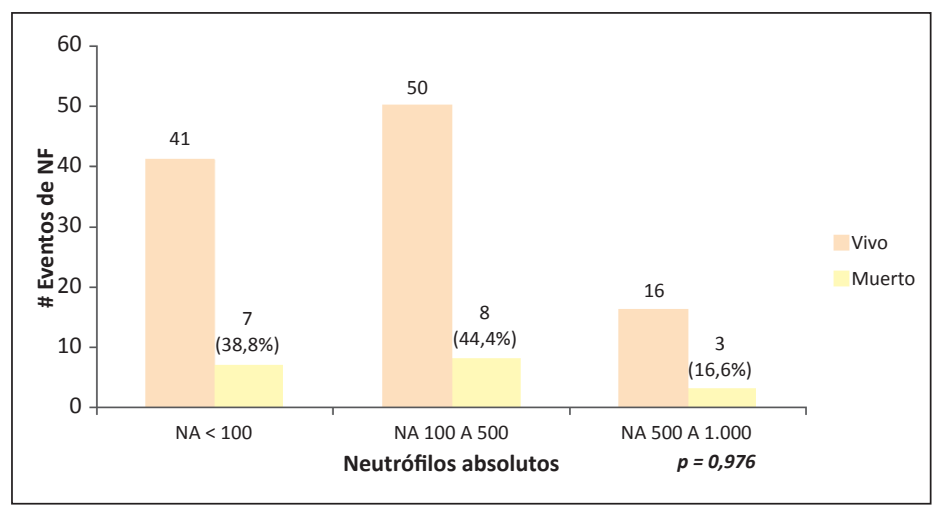

Figura 2. Asociación entre neutrófilos absolutos y mortalidad en 125 pacientes con neutropenia febril que recibieron cefepima.

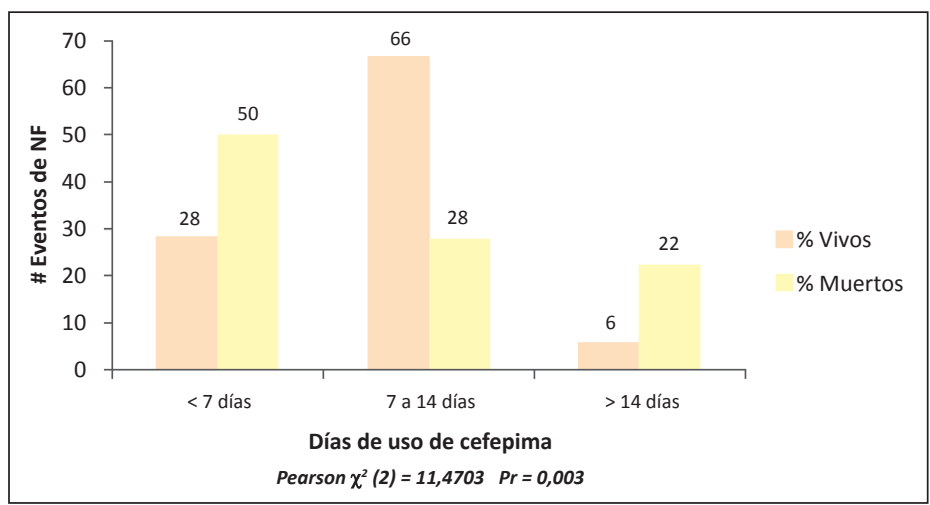

Figura 4. Asociación entre mortalidad y días de uso de cefepima en 125 pacientes con neutropenia febril.

\section{Microorganismos aislados}

De los cultivos que resultaron positivos se tipificaron bacterias en $96 \%$ y hongos en $4 \%$. La frecuencia porcentual de aislados microbiológicos fue: Escherichia coli 29,6\%; Staphylococcus aureus 12,3\%; Klebsiella pneumoniae 11,1\%; Pseudomonas aeruginosa 9,8\%; otras enterobacterias 9,8\% y Staphylococcus coagulasa negativa $19,7 \%$ (Figura 1 ).

\section{Uso de cefepima}

Recibieron cobertura con cefepima entre 7 y 14 días $60,8 \%$, por menos de 7 días $31,2 \%$ y por más de 14 días $8 \%$. El $57 \%$ de todas las NF recibió otro antimicrobiano adicional a cefepima. Dentro de la cobertura antimicrobiana asociada con el uso de cefepima se encontró que $28 \%$ de las NF recibieron concomitantemente vancomicina, $15,2 \%$ anfotericina $\mathrm{B}, 13,6 \%$ fluconazol, $9,6 \%$ albendazol y $8,8 \%$ metronidazol. Se realizó cambio de antimicrobiano a meropenem en $8 \%$, a piperacilina/tazobactam en $4 \%$ y a imipenem en $1,6 \%$.

En las Figuras 2 a 5 se describen las principales características clínicas del total de los pacientes con NF en manejo con cefepima y la asociación de mortalidad con

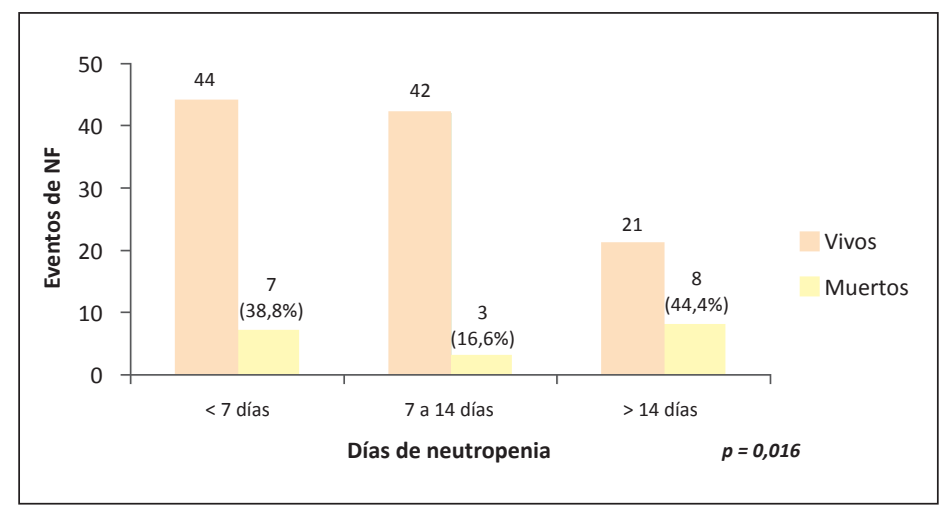

Figura 3. Asociación entre mortalidad y días de neutropenia en 125 pacientes tratados con cefepima.

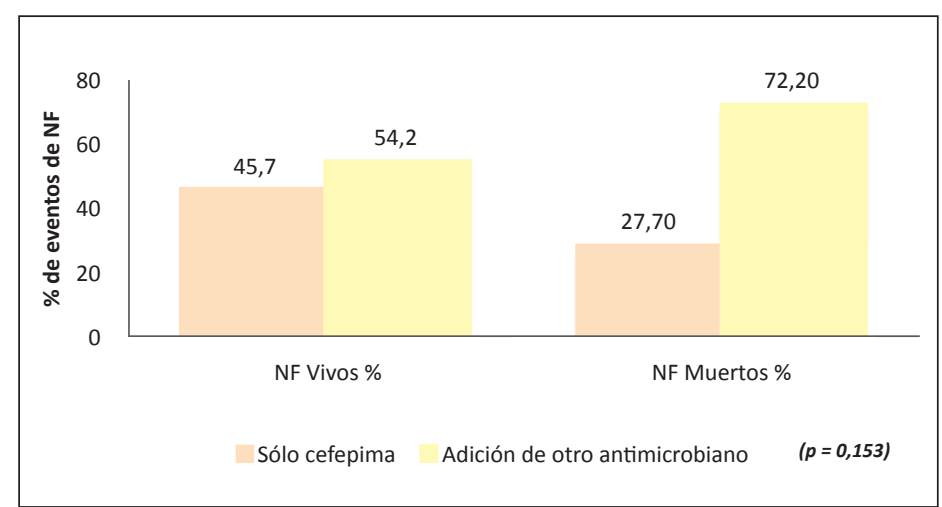

Figura 5. Relación entre el uso de otros antimicrobianos adicionales y la mortalidad en 125 pacientes con neutropenia febril que recibieron cefepima. 
respecto al recuento de neutrófilos absolutos, duración total de neutropenia, duración en días del uso de cefepima y uso concomitantemente de otro antimicrobiano respectivamente.

\section{Pacientes fallecidos}

De los pacientes fallecidos, $61 \%$ eran mujeres $(\mathrm{p}=0,637), 100 \%$ tenía de base una neoplasia hematológica $(\mathrm{p}=0,0085)$, ninguno de ellos tenían profilaxis antimicrobiana, $27,7 \%$ tuvo manejo previo con factor estimulante del crecimiento de colonias $(\mathrm{p}=0,267)$ y $50 \%$ tenía una o más co-morbilidades $(p=0,432)$. En $22 \%$ no se documentó un foco infeccioso $(\mathrm{p}=0,432)$ y en los que se confirmó proceso infeccioso, la frecuencia de hemocultivos positivos fue en $65 \%$. Los aislados microbiológicos más frecuentes en las bacteriemias fueron $E$. coli $36 \%$; Pseudomonas spp y $S$. aureus en $18 \%$ cada uno (Figura 6). En ninguno se documentó hongos ni parásitos. En la Figura 7 se describe la susceptibilidad microbiológica a la cefepima en los pacientes que fallecieron.

\section{Discusión}

En la literatura científica está reportada la mortalidad general de la NF entre 6 y $20 \%{ }^{19,25,26}$; y está descrito que los factores de riesgo más importantes para su presentación incluyen edad avanzada, co-morbilidades, tipo de cáncer, número y clase de agentes mielo-supresores ${ }^{7}$. La $\mathrm{NF}$ es una complicación potencialmente fatal y costosa en pacientes que se encuentran en quimioterapia para cáncer ${ }^{27}$

En los tres meta-análisis mencionados al inicio, la mortalidad aumentó con el uso de cefepima, en comparación con la de otros -lactámicos, ponderadas en 26 y $44 \%{ }^{16,17,19}$ lo cual puso en duda la seguridad de este medicamento en esta entidad clínica. Sin embargo, la FDA realizó un nuevo meta-análisis ${ }^{22}$, que incluyó 50 estudios adicionales (776 pacientes), que no fueron incluidos en los meta-análisis publicados por Dafna Yahav, Mical Paul y cols.; se evalúo el uso de cefepima vs otros -lactámicos, con una mortalidad general a 30 días de 7,8\% para los pacientes tratados con cefepima $v s 6,5 \%$ para los comparadores (IC: 95\% 9,22-45,42), resultados que no alcanzan significancia estadística para corroborar que hay un aumento de la mortalidad con el uso de cefepima ${ }^{28,29,12}$. Es necesario resaltar que en el análisis de este estudio los autores reconocen que los datos no favorecen a cefepima; no obstante, el análisis por subgrupo (NF) debe realizarse con precaución debido al pequeño número de pacientes y pocas muertes como desenlace ${ }^{22}$.

En nuestro estudio, en el que se incluyeron 125 eventos de NF, número equiparable al de muchos de los estudios incluidos en los meta-análisis antes mencionados, se

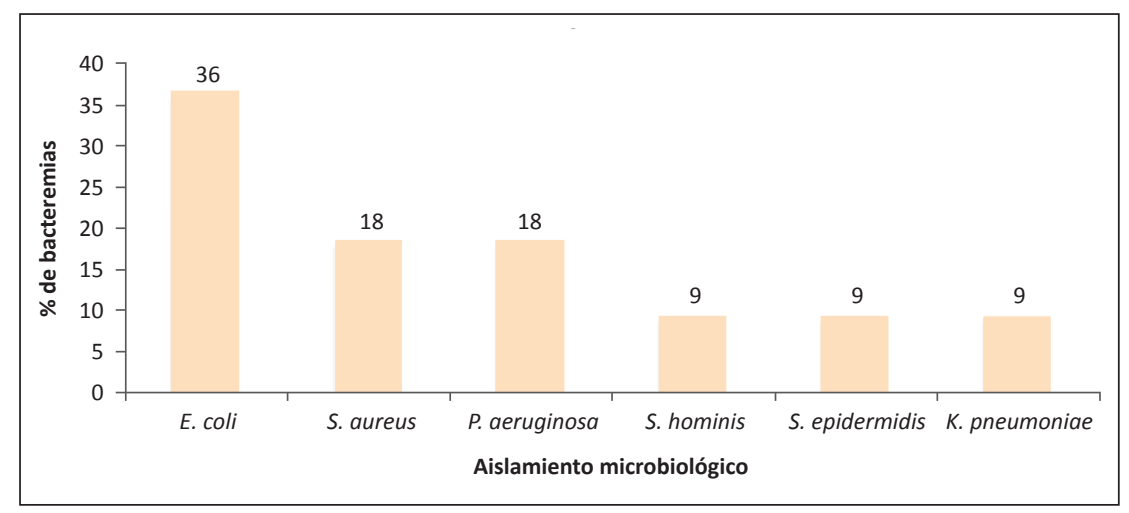

Figura 6. Asociación entre el aislamiento microbiológico en bacteriemias y mortalidad en 125 pacientes con neutropenia febril que recibieron cefepima.

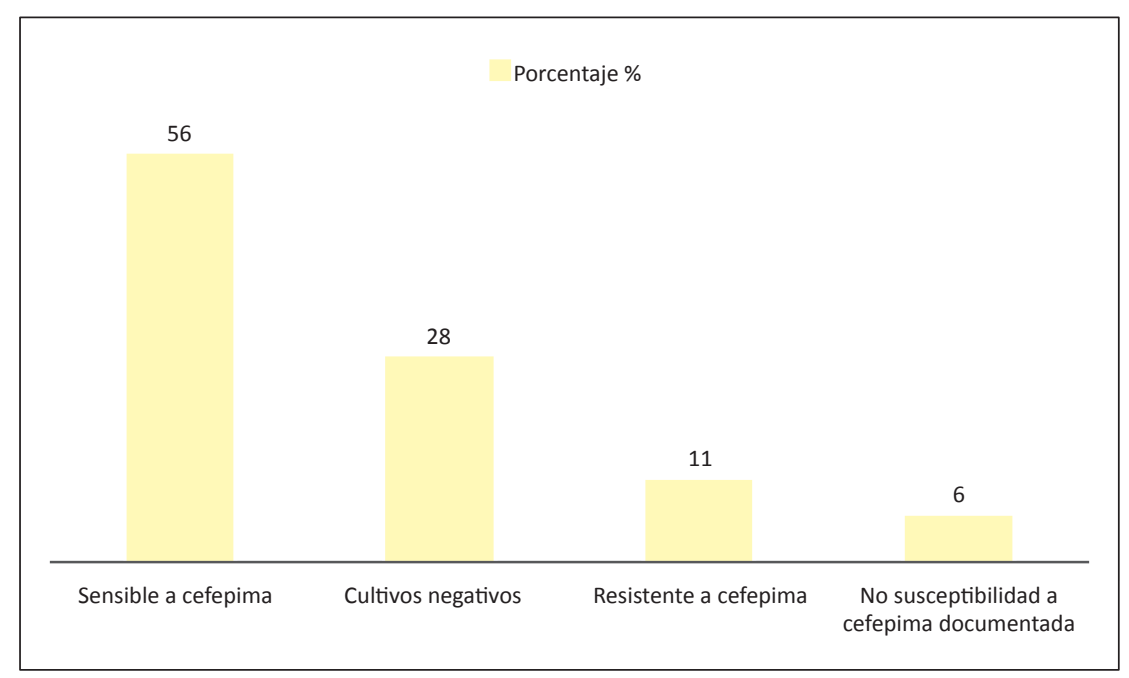

Figura 7. Sensibilidad de cultivos a cefepima en los pacientes que fallecieron.

observó mortalidad de 14,4\% con uso de cefepima, comparable con la mortalidad general descrita en NF. Como limitaciones del presente estudio encontramos que no se conoce la mortalidad general de NF para este mismo período de tiempo en nuestra institución, y tampoco se dispone de un comparador de los desenlaces de NF con otro antimicrobiano, esto probablemente debido a que la frecuencia de uso de otros antimicrobianos como primera elección se restringía a indicaciones muy precisas y limitadas según el protocolo.

Recientemente, Al-Hasan MN y cols., en una cohorte retrospectiva evaluaron la efectividad del tratamiento con cefepima y encontraron una mortalidad de $15 \%$ a 28 días, en pacientes con bacteriemias por bacilos gramnegativos similar al de otros comparadores -lactámicos ${ }^{29,30}$.

Vale la pena resaltar que la duración de la neutropenia en días es proporcional a la mortalidad en nuestro estudio; sin embargo, no se puede diferenciar si la causa de 
la mortalidad es derivada del uso de cefepima, efectos adversos de ésta o del tiempo de neutropenia, pero sí está establecido en la literatura médica mundial que la duración de la neutropenia se correlaciona con mortalidad, en forma independiente del antimicrobiano empleado ${ }^{30,31}$. Igualmente, es importante destacar que la mitad de los pacientes que fallecieron no alcanzaron una duración mayor de siete días de tratamiento lo cual puede estar relacionado con la gravedad del cuadro infeccioso inicial; no se puede diferenciar si esta mortalidad se relaciona con el uso de cefepima, sus efectos adversos o la presencia de un microorganismo inadecuadamente cubierto por el antimicrobiano.

De todas las muertes con NF y cefepima, todas tenían neoplasia hematológica como condición predisponente, lo que puede sugerir que la presencia de ésta puede estar asociada con mayor riesgo de mortalidad. Sin embargo, hay que aclarar que el número de pacientes con neoplasia hematológica vs no hematológica (tumor sólido, SIDA y trasplante) es mucho mayor y no es posible establecer una comparación entre ambos grupos. Adicionalmente, los esquemas de quimioterapia en neoplasias hematológicas condicionan mayor tiempo y gravedad de aplasia medular, por lo tanto no se puede diferenciar si el riesgo de mortalidad es debido a la presencia de dicha neoplasia o a su tratamiento quimioterapéutico ${ }^{31,32}$. Towne y cols., al realizar un nuevo análisis de la causa de mortalidad en pacientes tratados con cefepima en NF encontraron que en $36 \%$ de un total de 74 casos, ésta estaba relacionada con la progresión de su enfermedad neoplásica ${ }^{32,33}$.

En países desarrollados se reportan como etiología de infección en pacientes con NF bacterias grampositivas $(60-70 \%)$, probablemente por la proliferación del uso de catéteres vasculares centrales ${ }^{12,19,23,24,29}$. Sin embargo, esta documentación microbiológica es altamente variable de acuerdo a la población, reportándose en países en desarrollo y en el Medio Oriente un predominio en bacterias gramnegativas $^{33-37}$. En nuestra institución se documentaron con mayor frecuencia bacterias gramnegativas en más de $50 \%$ de todos los eventos de NF y en $63 \%$ de los que fallecieron, sin poderse establecer una correlación entre la etiología y la mortalidad. Es importante mencionar, que está descrito en la literatura científica, que la presentación de bacteriemia por bacilos gramnegativos conlleva un mayor riesgo de mortalidad, en especial las infecciones causadas por $P$. aeruginosa $a^{23,38,37}$, lo cual podría tener un impacto en la mortalidad en nuestra población dado que en un porcentaje importante (18\%) de todos los casos de NF se confirmó bacteriemia y de estos en $65 \%$ fue por bacilos gramnegativos.

En este estudio se documentó falla terapéutica en 24\% de los casos; no obstante, en el meta-análisis más reciente de M. Paul y cols. ${ }^{19}$, los autores definieron como falla terapéutica a la falla clínica, microbiológica, eventos adversos y a la sobre-infección bacteriana, sin evidenciar una desventaja con el uso de cefepima y sin alcanzar la significancia estadística. Adicionalmente, es importante mencionar que los autores reconocen que evaluar un criterio como falla terapéutica, especialmente en lo que respecta a falla clínica es propenso a sesgos, dada la alta frecuencia de fiebre prolongada en pacientes neutropénicos, al uso de otros antimicrobianos concomitantemente al esquema empírico inicial y/o a la modificación del esquema antimicrobiano y a la ausencia de correlación entre falla terapéutica y mortalidad en todos los estudios incluidos en el meta-análisis ${ }^{19}$.

El 50\% de los pacientes que fallecieron tenía co-morbilidades, con un espectro heterogéneo de enfermedades que no permitió detectar asociaciones con el desenlace por el bajo tamaño de muestra. Sin embargo, está descrito en la literatura científica que la presencia de enfermedad pulmonar obstructiva crónica (EPOC) exacerbada, en manejo con broncodilatadores y/o corticoesteroides, constituye un factor de riesgo para infección grave y mortalidad de acuerdo a la escala MASCC (escala de la Asociación Multinacional de Tratamiento de Soporte en Cáncer); adicionalmente como limitante en nuestro estudio, no disponemos de todos los datos clínicos al inicio del cuadro referentes a problemas médicos activos como son falla hepática, mucositis significativa, síntomas gastrointestinales graves o cambios neurológicos que constituyen criterios de alto riesgo para infección grave y de muerte ${ }^{23}$. En nuestra muestra de pacientes con NF sólo se documentaron dos casos con EPOC y ninguno de estos pacientes falleció.

\section{Conclusiones}

Según lo reportado en la literatura médica, a pesar que Peter W. Kim y cols. ${ }^{22}$, con los hallazgos de su metaanálisis aclaran el panorama sobre la seguridad del uso de cefepima en NF, debe tenerse en cuenta que debido al bajo número de eventos de NF y mortalidad reportado en los estudios, las conclusiones que se tomen en términos de mortalidad y uso de cefepima deben interpretarse con precaución y aún se requieren estudios prospectivos analíticos, controlados, que permitan dilucidar el papel de cefepima en cada uno de los escenarios clínicos propuestos, especialmente en NF. La mortalidad encontrada en este estudio es similar a la mortalidad general en NF reportada en la literatura médica permitiendo que este medicamento continúe siendo prescrito en nuestra institución para esta indicación. Por ahora, en las guías de la Sociedad Americana de Enfermedades Infecciosas publicadas en 2010 se recomienda la monoterapia con -lactámicos en las cuales se incluye el uso de cefepima como recomendación A y nivel de evidencia $\mathrm{I}^{23}$. 


\section{Resumen}

Introducción: La eficacia de cefepima en pacientes adultos con cáncer y neutropenia febril (NF) es objeto de controversia en las publicaciones científicas. Objetivo: Describir las características demográficas y la mortalidad general de los pacientes adultos con NF tratados con cefepima en un hospital latinoamericano de cuarto nivel. Pacientes y Métodos. Estudio observacional descriptivo, de corte transversal en el que se incluyeron todos los pacientes tratados con cefepima en el Hospital Universitario
San Ignacio de Bogotá, Colombia entre enero de 2004 y diciembre de 2008. Resultados: Recibieron cefepime un total 333 pacientes, de los cuales 125 tenían diagnóstico de NF y cumplían criterios pre-establecidos de inclusión y exclusión. Como desenlace final se encontró una mortalidad de $14,4 \%$, un porcentaje similar a la mortalidad general en NF reportada en la literatura médica. Conclusiones: Aún no hay claridad sobre la eficacia del uso de cefepima en NF; sin embargo, tampoco se ha proscrito su uso y los datos del presente estudio no encontraron un riesgo adicional de mortalidad.

\section{Referencias bibliográficas}

1.- Pizzo P A. Fever in immunocompromised patients. N Engl J Med 1999; 341 (12): 893-900.

2.- Antoniadou A, Giamarellou H. Fever of unknown origin in febrile leucopenia. Infect Dis Clin North Am 2007; 1055-90.

3.- Giamarellou H, Antoniadou A. Infectious complications of febrile leucopenia. Infect Dis Clin North Am 2001; 15 (2): 457-82.

4.- Giamarellos E J, Grecka P, Poulakou G, Anargyrou K, Katsilambros N, Giamarellou H. Assessment of procalcitonin as a diagnostic marker of underlying infection in patients with febrile neutropenia. Clin Infect Dis 2001; 32 (12): 1718-25.

5.- Jimeno A, Garia-Velasco A, del Val O, González-Billalabeitia E, Hernando S, Hernández R, et al. Assessment of procalcitonin as a diagnostic and prognostic marker in patients with solid tumors and febrile neutropenia. Cancer 2004; 100 (11): 2462-9.

6.- Calandra T, Cohen J. The International Sepsis Forum Consensus Conference on Definitions of Infection in the Intensive Care Unit for the International Sepsis Forum Definition of Infection in the ICU Consensus Conference Crit. Care Med 2005; 33 (7): 1538-48. Review.

7.- Kuderer N M, Dale D C, Crawford J, Cosler L E, Lyman G H. Mortality, morbidity, and cost associated with febrile neutropenia in adult, cancer patients. Cancer 2006; 106: 2258-66.

8.- Pizzo P A. Management of fever in patients with cancer and treatment induced neutropenia. N Engl J Med 1993; 328 (18): 1323-32.

9.- Cornely O A, Reichert D, Buchheidt D, Maschmeyer G, Wilhelm M, Chiel X, et al. Three-armed multicenter randomized study on the empiric treatment of neutropenic fever in a high risk patient population (PEG study III). Proceeding of the 41 st Interscience Conference on Antimicrobial Agents and Chemotherapy; Chicago, IL December 16-19; 2001: Abstr. 446.

10.- Cornely O A, Bethe U, Seifert H, Breuer K, Schütt-Gerowitt H, Salzberger B, et al.
A randomized monocentric trial in febrile neutropenic patients: ceftriaxone and gentamicin vs. cefepime and gentamicin. Ann Hematology 2002; 81: 37-43.

11.- Noskin G A, Vesole D, Szer J, Sánchez S, Bow E J, Laverdie M, et al. Multicenter efficacy and safety study of piperacillin/tazobactam as empiric treatment for neutropenic fever in patients with hematologic malignancies. In: Abstracts of the Annual Meeting of the Infectious Diseases Society of America, San Diego, CA, 2003. Infectious Diseases Society of America, Alexandria, VA, USA. Abstract no. 373.

12.- Bow E J, Rotstein C, Noskin G A, Laverdiere M, Schwarer A P, Segal BH, et al. A randomized, open-label, multicenter comparative study of the efficacy and safety of piperacillin-tazobactam and cefepime for the empirical treatment of febrile neutropenic episodes in patients with hematologic malignancies. Clin Infect Dis 2006; 43 (4): 447-59. Epub 2006 Jul 10.

13.- Glauser M, Dekker A W, Van Marwijk-Kooy M, et al. A multicenter comparative study of cefepime $v s$ ceftazidime as empiric therapy in the treatment of febrile episodes of neutropenic patients. Joint clinical/statistical review of NDA 50,679/SE1-002. http://www. fda.gov/cder/foi/nda/97/050679s002.htm (accedido el 2 de diciembre de 2008).

14.- Thirumala R, Ramaswamy M, Chawla S. Diagnosis and management of infectious complications in critically ill patients with cancer. Crit Care Clin 2010; 26 (1): 59-91.

15.- Williams K. Why monotherapy? J Antimicrob Chemother 2009, 63, Suppl. 1, i18-i20.

16.- Paul M, Yahav D, Fraser A, Leibovici L. Empirical antibiotic monotherapy for febrile neutropenia: systematic review and metaanalysis of randomized controlled trials. J Antimicrob Chemother 2006; 57: 176-89.

17.- Yahav D, Paul M, Fraser A, Sarid N, Leibovici L. Efficacy and safety of: a systematic review and meta-analysis Lancet Infect Dis 2007; 7: 338-48.
18.- Álvarez C A, Ruiz A, Gil F, Ortiz G, Londoño D, Clavijo W, et al. Efficacy of cefepime continuous infusion versus an intermittent dosing regimen in adult critically ill patients. Randomized Clinical Trial. NCT00609375 (abstract K-4097) (http://www. mindcull.com/data/american-society-formicrobiology/icaac-2008-antimicrobial-agentsand-chemotherapy/).

19.- Paul M, Yahav D, Bivas A, Fraser A, Leibovici L. Anti-pseudomonal beta-lactams for the initial, empirical, treatment of febrile neutropenia: comparison of beta-lactams. Cochrane Database Syst Rev 2010; (11): CD005197.

20.- Zanetti G, Bally F, Greub G, Garbino J, Kinge T, Lew D, et al. Cefepime Study Group. Cefepime versus imipenem cilastatin for treatment of nosocomial pneumonia in intensive care unit patients: a multicenter, evaluator-blind, prospective, randomized study. Antimicrobial Agents Chemotherapy 2003; 47 : 3442-7.

21.- Raad I, Escalante C, Hachem R Y, Hanna H A, Husni R, Afif C, et al. Treatment of febrile neutropenia patients with cancer who require hospitalization: a prospective randomized study comparing imipenem and cefepime. Cancer 2003; 98: 1039-47.

22.- Kim P, Wu Y, Cooper Ch, Rochester G, Valappil T, Wang Y, et al. Meta-analysis of a possible signal of increased mortality associated with cefepime use. Clin Infect Dis 2010; 51 (4): 381-9.

23.- Freifeld A, Bow E, Sepkowitz K, Boeckh M, Ito J, Mullen C, et al. Clinical practice guideline for the use of antimicrobial agents in neutropenic patients with cancer: 2010 Update by the Infectious Diseases Society of America. Clin Infect Dis 2011; 52 (4): e56-93.

24.- Hughes W T, Armstrong D, Bodey G P, Bow E J, Brown A E, Calandra T, et al. 2002 guidelines for the use of antimicrobial agents in neutropenic patients with cancer. Clin Infect Dis 2002; 34: 730-51.

25.- Elting L S, Rubenstein E B, Rolston K V, Bodey G P. Outcomes of bacteremia in 
patients with cancer and neutropenia: observations from 2 decades of epidemiological and clinical trials. Clin Infect Dis 1997; 25: 247-59.

26.- Segal B H, Freifeld A G, Baden L R, Brown A E, Casper C, Dubberke E, et al. Prevention and treatment of cancer-related infections. J Natl Compr Canc Netw 2008; 6: 122-74.

27.- Lyman G H, Michels S L, Reynolds M W, Barron R, Tomic K S, Yu J. Risk of mortality in patients with cancer who experience febrile neutropenia. Cancer 2010; 116 (23): 5555-63.

28.- Cherif H, Bjorkholm M, Engervall $P$, Johansson P, Ljungman P, Hast R, et al. A prospective, randomized study comparing cefepime and imipenem cilastatin in the empirical treatment of febrile neutropenia in patients treated for hematological malignancies.
Scand J Infect Dis 2004; 36: 593-600.

29.- Al-Hasan M N, Eckel-Passow J E, Baddour L M. Cefepime effectiveness in Gram-negative bloodstream infections. J Antimicrob Chemother 2011; 66 (5): 1156-60.

30.- Rolston K V. Prediction of neutropenia. Int J Antimicrob Agents 2000; 16 (2):113-5.

31.- Scott S. Identification of cancer patients at high risk of febrile neutropenia. Am J Health-Syst. Pharm 2002; 59 (Supple 4): S16-9.

32.- Towne T G, Lewis J S, Echevarria K. Efficacy and safety of cefepime. Lancet Infect Dis 2009; 9: 4-6.

33.- Baskaran N D, Gan G G, Adeeba K, Sam I C. Bacteremia in patients with febrile neutropenia after chemotherapy at a university medical center in Malaysia. Int J Infect Dis 2007; 11 (6): 513-7.

34.- Kanafani Z A, Dakdouki G K, Chammas K I,
Eid S, Araj G F, Kanj S. Bloodstream infections in febrile neutropenic patients at a tertiary care center in Lebanon: a view of the past decade. Int J Infect Dis 2007; 11 (5): 450-3.

35.- Paul M, Gafter G A, Leibovici L, Bishara J, Levy I, Yaniv I, et al. The epidemiology of bacteremia with febrile neutropenia: experience from a single center, 1988-2004. Israel Med Assoc J 2007; 9 (6): 424-9.

36.- Chen C Y, Tsay W, Tang J L, Tien H F, Chen Y C, Chang S C, et al. Epidemiology of bloodstream infections in patients with haematological malignancies with and without neutropenia. Epidemiol Infect 2010; 138 (7): 1044-51.

37.- Klastersky J, Ameye L, Maertens J, Georgala A, Muanza F, Aoun M, et al. Bacteraemia in febrile neutropenic cancer patients. Int J Antimicrob Agents 2007; 30 (Suppl 1): S51-9. 